\section{Concurrent C: Workshop: Family Violence 11 BROKERING INFORMATION TO SUPPORT FAMILY VIOLENCE PREVENTION AND INTERVENTION EFFORTS IN NEW ZEALAND}

doi:10.1136/injuryprev-2012-040580f.11

J Fanslow*, R Dixon, N Paton, G Richards*, P Gulliver. New Zealand Family Violence Clearinghouse, University of Auckland, New Zealand

Background Access to high quality information is an essential feature of evidence informed practice in family violence prevention and intervention work.

Aim The New Zealand Family Violence Clearinghouse is the national centre for collating and disseminating information about domestic and family violence in Aotearoa.

Methods Collection takes place through review of international websites, scans of the academic literature, and outreach with NZ $\&$ international academics and practitioners. Collation and dissemination takes place through the NZFVC Library (electronic and physical), a monthly newsletter, customised searches by the information specialist and the production and publication of fact sheets, and issues papers. Active community engagement helps to identify common information needs across the sector.

Results Information dissemination: In the period 11/10/12-20/2/ 12 the website had 12395 visits by 8262 unique visitors from 109 countries (83\% from NZ). The newsletter is distributed to 1460 people, with the mailing list growing by 567 in 6 months. Two issues papers and multiple factsheets have been produced.

Community engagement includes developing and maintaining active relationships with a wide range of practitioners, policy makers, and researchers through attendance at community and national hui, speaking at events, individual meetings, phone and email contact.

Significance The Clearinghouse contributes to the family violence sector in NZ by acting as an information hub. By serving a wide audience, we play a significant role in identifying high quality information, making it accessible, 'funnelling' information to those who need it, identifying information gaps, and actively strategising on ways to meet these needs. abuse through early identification, assessment and referral of victims presenting to health services. The programme is premised on standardised system models of organisational change and reflects an indigenous whanau ora approach. Programme components include national guidelines, coordinators, technical advice, national networking, training, resources and evaluation.

Aim To describe the respective contributions of funders, national programme manager and researcher to the achievements of VIP within the health sector.

Methods National programme management and evaluation focuses on a coordinated approach to quality improvement. An external evaluation monitored development of VIP from 2004-2011 in 20 District Health Boards. The evaluation applied partner abuse (PA) and child abuse and neglect (CAN) programme audit tools measuring system indicators.

Results VIP scores increased incrementally over the six audit periods. Median audit score (possible range 0 to 100) increased from 20 to 74 for responsiveness to PA and from 37 to 81 for responsiveness to CAN. At the most recent audit (2011), 17 DHBs $(85 \%)$ achieved the benchmark target score $(>70)$ in both PA and CAN programmes, exceeding Ministry of Health expectations.

Significance/Contribution to the Field Longitudinal evaluations of national PA and CAN intervention programmes in health are limited. VIP design and external evaluation results provide evidence to guide future programme development. Recommended areas include transitioning programme evaluation from indicator compliance to a culture of continuous performance improvement and increasing provision of service delivery to families at risk. 